\title{
Amino acid transport in the unfertilized and fertilized mouse egg
}

\author{
S. R. M. Holmberg and M. H. Johnson \\ Department of Anatomy, University of Cambridge, Downing Street, \\ Cambridge CB2 3DY, UK.
}

\begin{abstract}
Summary. The transport of methionine into unfertilized and fertilized mouse eggs appears to involve active transport mechanisms with similar $V_{\max }, K_{\mathrm{m}}$, substrate specificity and independence from $\mathrm{Na}^{+}$. An exchange diffusion system with a similar amino acid specificity to the uptake system has also been found in both types of egg. An estimate of $6.5 \mathrm{fmol}$ has been made for the size of the total internal pool of exchangeable amino acids.
\end{abstract}

\section{Introduction}

The rates at which radioactive amino acids are incorporated into protein differ at different embryonic stages (Epstein \& Smith, 1973) and under different conditions at the same stage (Braude, 1979). Whilst these variations in incorporation may reflect genuine alterations in protein synthetic activity, they could also result from changes in uptake kinetics, endogenous pool size and specific activity, or from reorganization of intracellular compartments (Brinster, Wiebold \& Brunner, 1976). Previous studies on preimplantation mouse embryos have attempted to reduce these problems in two ways. (1) High exogenous concentrations of amino acid have been used in an attempt to swamp endogenous pools and bring the specific activity of the internal amino acid to the same level as that measured externally (Epstein \& Smith, 1973). (2) Brinster $e t$ al. (1976) measured intracellular specific activity directly and showed that it was difficult to expand endogenous pools so as to stabilize internal specific activity even at relatively high (10 mM) concentrations of external amino acid. These workers estimated total free intracellular leucine by labelling it with $\left[{ }^{14} \mathrm{C}\right] 1$-fluoro-2,4-dinitrobenzene and measured $\left[{ }^{3} \mathrm{H}\right]$ leucine taken up into the cells by active transport. From these figures it was possible to calculate not only intracellular specific activity but also endogenous pool size. Both were found to vary for embryos of different stages.

These two approaches have assumed that amino acids taken up by the cell are freely distributed internally into a pool which is directly drawn on for protein synthesis. We report here the results of an investigation into amino acid transport in unfertilized and fertilized mouse eggs which suggest that this assumption may not be valid.

\section{Materials and Methods}

\section{Superovulation and egg handling}

Random bred CFLP mice (Anglia Laboratories), 6-8 weeks of age, were induced to superovulate with 7.5 i.u. PMSG (Folligon: Intervet, Bar Hill, Cambridge, U.K.) intraperitoneally, followed $44-48 \mathrm{~h}$ later by 7.5 i.u. hCG (Chorulon: Intervet). Some females were paired with males after the hCG injection, and those with vaginal plugs $14 \mathrm{~h}$ later were recorded as mated. Mated and non-mated females were killed by cervical dislocation 14-15 h after the 
hCG injection. The oviducts were removed and the egg masses released into phosphate-buffered medium (PB1) (Whittingham, 1971) containing $0.4 \%(\mathrm{w} / \mathrm{v})$ bovine serum albumin.

The eggs were completely freed from cumulus cells by brief exposure to hyaluronidase (1 $\mathrm{mg} / \mathrm{ml}$ in PBS containing $20 \mathrm{mg}$ polyvinylpyrrolidone $/ \mathrm{ml}$ ). In pilot experiments it was shown that the presence or absence of the zona pellucida did not affect uptake kinetics and therefore zonae were retained throughout. The eggs were then allowed to recover in PB $1+\mathrm{BSA}$ for at least $30 \mathrm{~min}$.

Eggs from mated animals were examined for the presence of 2 pronuclei and a second polar body. Unfertilized eggs from mated females were discarded.

\section{Labelling procedures}

Preliminary studies to determine the most suitable macromolecule for the incubation medium indicated that fetal calf serum substantially reduced uptake of tracer compared to bovine serum albumin (BSA) or Ficoll (mol. wt. 400 000, Pharmacia, Uppsala, Sweden). Ficoll consistently gave uptake values about $1 \frac{1}{2}$ times those observed with BSA (dialysed or non-dialysed). This result appeared to be due to active stimulation by Ficoll rather than depression by BSA, since when both were present the uptake values corresponded to those of Ficoll. Ficoll also made egg handling difficult. Throughout these studies, therefore, $0.4 \%$ BSA was used.

Incubations were performed in $100 \mu \mathrm{l}$ PB1 $+0.4 \%$ BSA with appropriate modifications as described in 'Results'. Preincubations were also performed, as indicated in the 'Results', for at least $30 \mathrm{~min}$. The radiolabelled $\mathrm{L}-\left[{ }^{35} \mathrm{~S}\right]$ methionine (sp. act. $\sim 1000 \mathrm{Ci} / \mathrm{mmol}$ : Radiochemical Centre, Amersham, England) was obtained as an aqueous solution $(5.37 \mathrm{mCi} / \mathrm{ml})$. It was mixed with unlabelled methionine in PB1 + BSA to produce solutions of the required methionine concentration. Samples of the culture media were taken and counted after dilution. For each experimental condition, eggs were handled in groups of 5 , and in an excess of medium such that external levels of methionine were never limiting (ratio of volumes $20000: 1$ ).

After incubation, the embryos were transferred to $0.5 \mathrm{ml} \mathrm{PB1}+\mathrm{BSA}$ and then through seven $150 \mu$ drops of unlabelled PB1 + BSA (see 'Results'). Samples of the terminal washing fluids were taken for counting to check for the complete removal of free radioactive methionine.

Washed eggs were either counted directly or, in some experiments, were prepared for TCA precipitation. These eggs were lysed in $20 \mu \mathrm{l}$ lysis buffer and frozen and thawed. The protein was co-precipitated with $20 \mu \mathrm{l} 0.1 \% \mathrm{BSA}$ by $160 \mu \mathrm{l} 10 \% \mathrm{TCA}$. After filtering, the filtrate samples and the filter papers were counted. With the incubation times and methionine concentrations used, incorporation into protein was negligible relative to total levels of methionine taken up.

\section{Counting procedures}

The numbers of counts per egg were related to the total amount of methionine, labelled and unlabelled, taken up by use of the formula:

$$
\mathrm{M}=\frac{(\mathrm{Ce}-\mathrm{Cw})}{\mathrm{Ci}} . \mathrm{Mi} . \mathrm{Vi}
$$

where $\mathrm{M}=$ mol methionine taken up; $\mathrm{Ce}=$ c.p.m. per egg; $\mathrm{Cw}=$ background c.p.m. in wash solution sample (in practice $\mathrm{Cw}$ was negligible and this term may be dropped from the equation); $\mathrm{Vi}=$ volume of incubation solution counted; $\mathrm{Mi}=$ molarity of incubation solution; $\mathrm{Ci}=\mathrm{c} . \mathrm{p} . \mathrm{m}$. in incubation solution sample.

\section{Data handling}

The counts from at least 5 eggs were pooled to obtain a mean value for methionine uptake at any given point. In experiments to calculate the initial rate of uptake, a linear regression 
programme was used to draw the best line through a set of points at 5 different times selected during pilot experiments to fall on the linear portion of an uptake-time graph. Student's $t$ test was used to assess significance levels. Each set of data represents pooled results from duplicate experiments unless otherwise indicated.

\section{Results}

The effect of the composition of the washing medium on the amino acid content of the fertilized and unfertilized eggs was tested and the results are shown in Text-fig. 1. In the absence of external methionine, the transported amino acid was not released from the egg at significant rates, but when methionine was present in the washing medium, radioactivity was lost rapidly. It seemed probable that an exchange system was operating, and this was confirmed by pre-equilibrating the eggs with $25 \mu \mathrm{M}$ unlabelled methionine and then transferring the eggs to a solution of $25 \mu \mathrm{m}$ labelled methionine. Labelled amino acid entered the eggs under these equilibrium conditions. In all further experiments, incubated eggs were washed and stored in methionine-free medium.

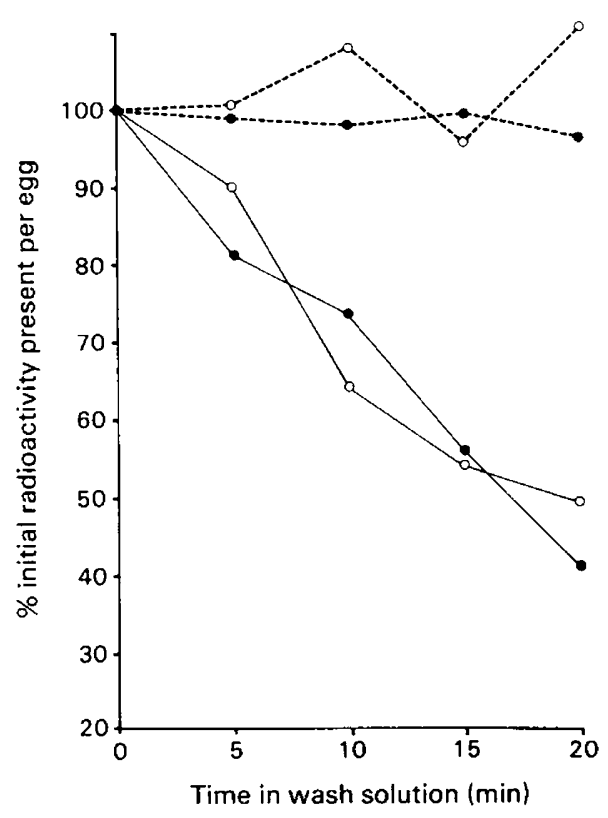

Text-fig. 1. Effect of washing conditions on amino acid content of mouse eggs. Eggs were incubated to equilibrium in $100 \mu \mathrm{M}$ radiolabelled methionine and then transferred to large volumes of wash solutions with or without $100 \mu \mathrm{M}$ unlabelled methionine. Groups of 5 eggs were sampled at intervals after the start of washing $(0 \mathrm{~min})$. The wash medium was changed at intervals, and sampled to ensure negligible accumulation of radioactivity. $(-\mathrm{O}-=$ fertilized eggs + methionine; $--=$ unfertilized eggs + methionine; $--0--=$ fertilized eggs without methionine; - - - unfertilized eggs without methionine). Results are pooled from triplicate experiments on both fertilized and unfertilized eggs. Similar results were obtained with methionine at concentrations of $50 \mu \mathrm{M}$ (1 exp.), $25 \mu \mathrm{M}$ (4 exps) and $10 \mu \mathrm{M}$ ( 2 exps).

The kinetics of uptake of methionine into unfertilized eggs were measured and compared to those of fertilized eggs. Net uptake ceased within $60 \mathrm{~min}$ of the start of the incubation (Text-fig. 2). Lineweaver-Burke plots of initial uptake rates against external methionine concentration (Text-fig. 3) gave values for $V_{\max }$ and $K_{\mathrm{m}}$ as recorded in Table 1. The net total uptake of 


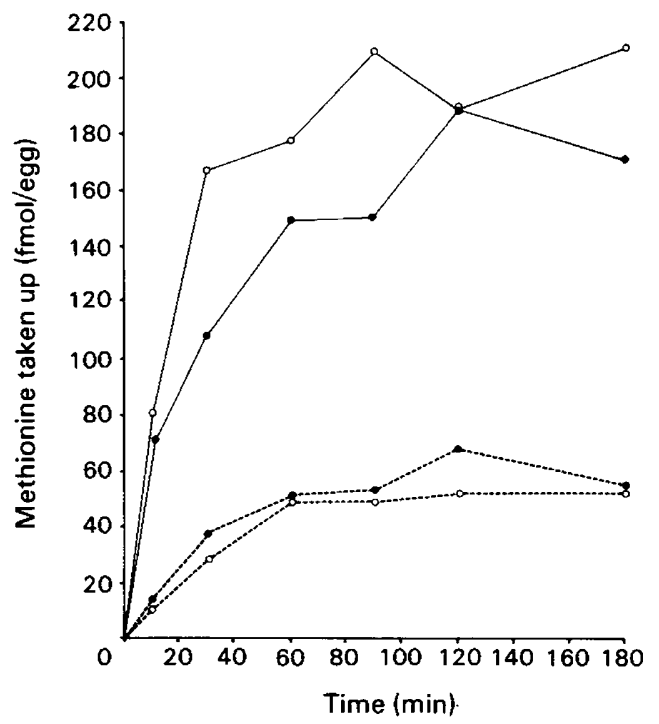

Text-fig. 2. Effect of incubation time on net uptake of amino acid into mouse eggs. Eggs were incubated in radiolabelled methionine at either 10 or $100 \mu \mathrm{M}$ concentration sampled in groups of 5 at various times, washed in methionine-free medium and counted. $--=$ fertilized, $100 \mu \mathrm{M}$ methionine; $-\mathrm{O}-=$ unfertilized, $100 \mu \mathrm{M}$ methionine; $----=$ fertilized, $10 \mu \mathrm{M}$ methionine; - - - - unfertilized, $10 \mu \mathrm{M}$ methionine. Results at each concentration are pooled from 3 experiments with fertilized eggs and from 4 experiments with unfertilized eggs. Additional experiments at $1,20,50$ and $250 \mu \mathrm{M}$ methionine are not plotted.

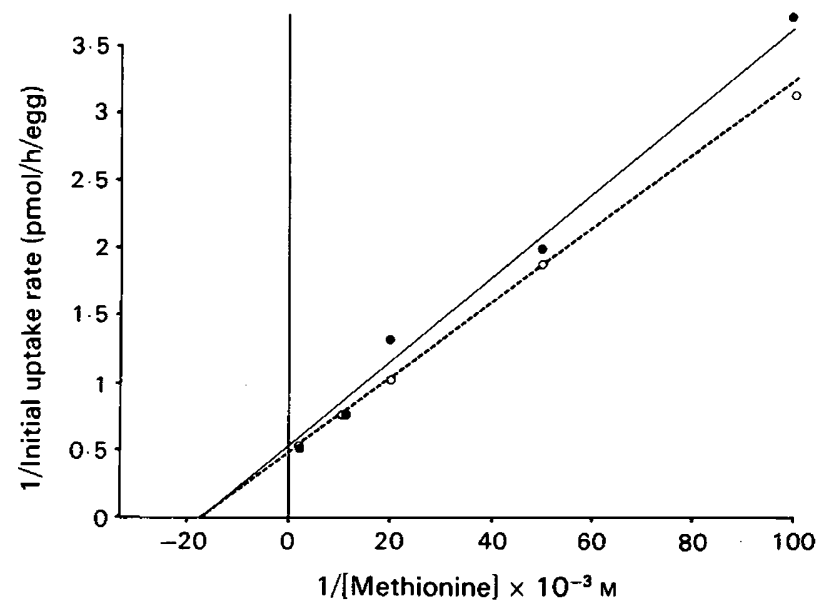

Text-fig. 3. Lineweaver-Burke plots of the reciprocals of initial uptake rates per egg (O, fertilized; $O$, unfertilized) against external methionine concentration. Data are pooled from 3 experiments. There is no statistical difference between the slopes of the two lines.

Table 1. Transport kinetics of methionine uptake into mouse eggs and early embryos

\begin{tabular}{lccc}
\hline & Unfertilized eggs & Fertilized eggs & 4-cell embryo* \\
\hline$V_{\max }(\mathrm{pmol} / \mathrm{embryo} / \mathrm{h})$ & 2.00 & 1.82 & 1.50 \\
$K_{\mathrm{m}}(\mu \mathrm{mol})$ & 56 & 57 & 62.5 \\
\hline
\end{tabular}

* Data taken from Borland \& Tasca (1974). 
methionine at the equilibrium position for each external concentration is recorded in Table 2 , together with calculations of the ratio of internal to external concentration of free methionine at equilibrium. These calculations were based on the assumption of even distribution through a cytoplasmic volume of $0.18 \mathrm{nl}$. It is evident that a net accumulation against gradient occurred at all external concentrations.

The specificity of the uptake transport mechanism did not appear to differ in fertilized and unfertilized eggs (Table 3) and was the same as the specificity of the exchange mechanism (Textfig. 4). The results of a number of experiments that established the nature of the carrier-mediated

Table 2. Equilibrium values for net uptake of methionine at different external methionine concentrations in unfertilized mouse eggs

\begin{tabular}{ccc}
\hline $\begin{array}{c}\text { External conc. } \\
(\mu \mathrm{M})\end{array}$ & $\begin{array}{c}\text { Net uptake } \\
(\mathrm{fmol} / \mathrm{egg})\end{array}$ & $\begin{array}{c}\text { Ratio of internal : external } \\
\text { methionine conc. }\end{array}$ \\
\hline 1 & $9 \cdot 6$ & 53 \\
10 & 46 & 26 \\
25 & 97 & 22 \\
50 & 141 & 16 \\
100 & 187 & 11 \\
\hline
\end{tabular}

Values represent the mean of 3 experiments.

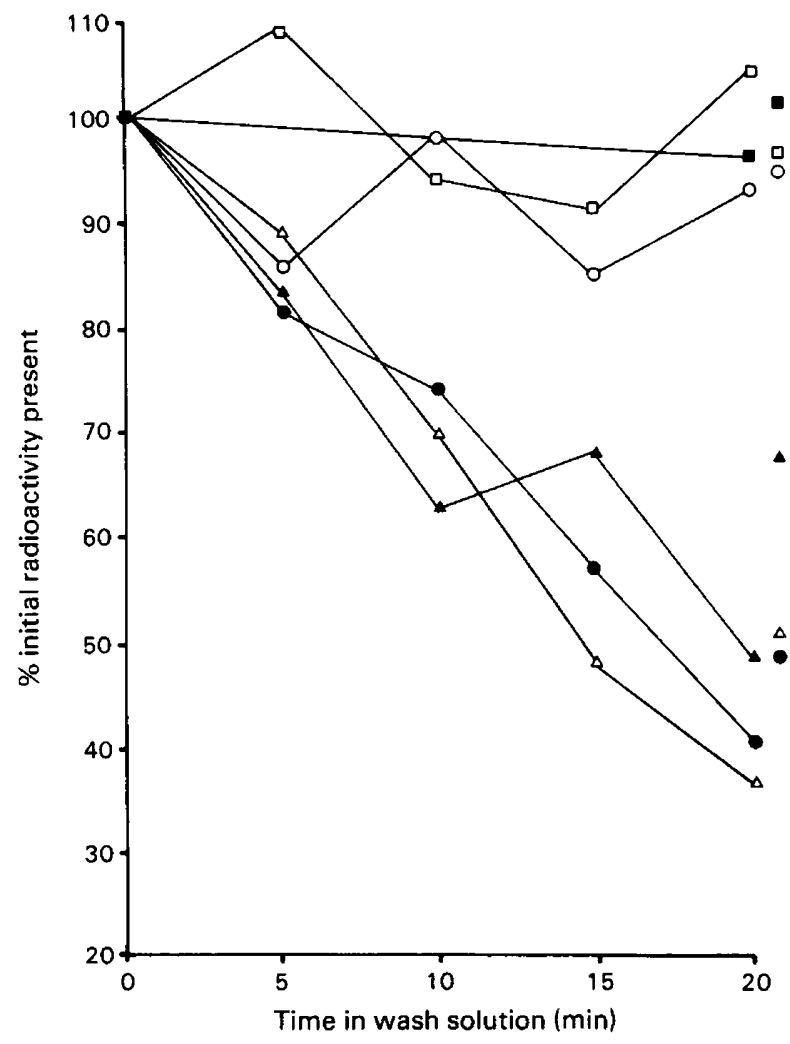

Text-fig. 4. Rate of radioactivity loss from unfertilized eggs incubated for $60 \mathrm{~min}$ in radiolabelled methionine $(100 \mu \mathrm{M})$ and then transferred to wash solutions containing $100 \mu \mathrm{M}$ unlabelled amino acids. The 20-min values for similarly treated fertilized eggs are shown on the right. $\square$, Glycine; $O$, alananine; $\mathbf{\square}$, no amino acid; $\Delta$, phenylalanine; $\Delta$, leucine; $\boldsymbol{O}$, methionine. 
transport system are summarized in Table 4. The system is $\mathrm{Na}$-independent and sensitive to azide. The partial inhibition in Ca-free medium was probably an indirect effect on the membrane rather than a reflection of calcium dependence.

Table 3. Effect of unlabelled amino acids in the incubation medium on initial rate of methionine uptake into eggs incubated in $10 \mu \mathrm{M}$-radiolabelled methionine

\begin{tabular}{lcc}
\hline & \multicolumn{2}{c}{ Rate (as \% of control values) } \\
\cline { 2 - 3 } Amino acid added & $\begin{array}{c}\text { Unfertilized } \\
\text { eggs }\end{array}$ & $\begin{array}{c}\text { Fertilized } \\
\text { eggs }\end{array}$ \\
\hline None & 100 & 100 \\
$50 \mu \mathrm{M}$-leucine & $14^{* *}$ & $15^{* *}$ \\
$50 \mu \mathrm{M}$-glycine & $117^{*}$ & $91^{*}$ \\
$50 \mu \mathrm{M}$-phenylalanine & $23^{* *}$ & $31^{* *}$ \\
$50 \mu \mathrm{M}$-alanine & $94^{*}$ & $85^{*}$ \\
\hline
\end{tabular}

${ }^{*} P>0.2 ;{ }^{* *} P<0.01$.

Table 4. Metabolic dependence of the rate of methionine uptake (\% of control values) into unfertilized eggs incubated in 10 or $20 \mu \mathrm{M}$ methionine at $37^{\circ} \mathrm{C}$

\begin{tabular}{llc}
\hline Preincubation medium & Incubation medium & Rate \\
\hline Control & Control & 100 \\
Control + 1 mM-ouabain & Control + 1 mM-ouabain & $111^{* \dagger}$ \\
Low sodium (25 mM) & Low sodium (25 mM) & $91^{* \dagger}$ \\
Calcium-free + 0.1\% EDTA & Calcium-free + 0.1\% EDTA & $55^{* *}$ \\
Calcium-free + 0.1\% EDTA & Control & $97^{*}$ \\
Control + 5 mM-azide & Control + 5 mM-azide & $45^{* *}$ \\
Control + 10 mM-fluoride & Control + 10 mM-fluoride & $93^{*}$ \\
\hline
\end{tabular}

Compared with control, ${ }^{*} P>0.2,{ }^{* *} P<0.01$.

† Compared with each other, $P>0 \cdot 2$.

\section{Discussion}

Three conclusions may be drawn from the results presented here. First, a carrier-mediated transport mechanism for amino acids operates in the mouse egg. Second, the bulk of the amino acid accumulated is available for exchange via a carrier-mediated amino acid exchange system; this will mean that experimental protocols which employ unlabelled amino acid in the washing medium may give erroneously low uptake values (Epstein \& Smith, 1973). Third, no major qualitative or quantitative differences are observed when fertilized and unfertilized eggs are compared.

A number of observations combine to support the existence of carrier-mediated transport of amino acids in the egg. The system satisfies Michaelis-Menten kinetics, appears to accumulate substrate against its gradient, saturates with increasing substrate concentration, is susceptible to competition and is dependent on metabolic processes. The transport system defined by these factors is almost identical kinetically to that previously described for the cleavage-stage mouse embryo (Borland \& Tasca, 1974, 1975) and shares with that system no obvious dependence on a 
$\mathrm{Na}^{+}$gradient to drive the amino acid transport. All embryonic stages from the prefertilization egg through cleavage also share many features with the L (leucine-preferring) system of the Erlich cell (Oxender \& Christiansen, 1963).

The evidence for an active concentration component in amino acid transport is less secure and derives principally from the values in Table 2 which show internal methionine to be present at equilibrium at greater concentration than levels in the external medium. This conclusion assumes that the apparent uphill transport of methionine is not due simply to exchange diffusion with internal endogenous pools of non-labelled exchangeable amino acids, i.e. methionine, leucine, phenylalanine and probably others. If the free endogenous leucine level of $119 \mathrm{fmol}$ calculated by Brinster et al. (1976) were to be available for exchange, then much of the methionine could be accumulated by exchange with this amino acid alone. Since equilibrium levels of methionine accumulation at external concentrations in excess of $100 \mu \mathrm{M}$ approach only $180 \mathrm{fmol}$, the exchange system appears to have the necessary capacity. However, this conclusion is misleading, since the methods used by Brinster et al. (1976) for estimating endogenous pool size ignore the possibility of exchange diffusion and were applied at times after such exchange would have been largely completed. Indeed, the value of $119 \mathrm{fmol}$ estimated by Brinster et al. (1976) represents non-precipitable leucine which is specifically not readily available for exchange. The implication of this conclusion is that two distinct pools of free amino acid may exist within the egg, one freely exchangeable with external methionine and the other only slowly exchangeable. The magnitude of the initial endogenous amino acid pool available for exchange with methionine is not known, but an estimate of it can be made by plotting the net uptake of methionine at equilibrium against the external concentration of methionine. The value of only $6.5 \mathrm{fmol}$ where the curve crosses the vertical axis may represent exchangeable amino acid at zero external concentration. This value is an order of magnitude lower than the nonexchangeable leucine pool described by Brinster et al. (1976). The foregoing considerations make it unlikely that the net uptake of methionine is mediated at the expense of internal exchangeable amino acids. An active accumulation against gradient is therefore implied, unless one is to assume further that the amino acid accumulated in this pool is not able to exert its full chemical potential, perhaps due to a division into two compartments in free equilibrium.

The kinetics of the exchange diffusion systems are in fact compatible with such a division, although they could equally be explained by independent effects of concentration on the opposing fluxes of the exchange mechanism. Thus, kinetically the exchange transport of amino acids across the egg membrane could be viewed as two enzymes working in opposite directions, such that

$$
\frac{\mathrm{dMi}}{\mathrm{dt}}=\mathrm{Ki} . \mathrm{Mo}-\mathrm{Ko} . \mathrm{Mi}
$$

where $\mathrm{Mi}$ and $\mathrm{Mo}$ are the total internal and external concentrations of free methionine respectively, Mo being constant, and $\mathrm{Ki}$ and $\mathrm{Ko}$ are the corresponding rate constants for influx and efflux.

Integrating (1) gives:

$$
\mathrm{Ki} \cdot \mathrm{Mo}-\mathrm{Ko} \cdot \mathrm{Mi}=\mathrm{Ki} \cdot \mathrm{Mo} \cdot \mathrm{e}^{-\mathrm{Ko} \cdot \mathrm{t}}
$$

Thus at a time $=\mathrm{t}$ during the incubation,

$$
\operatorname{Mi}(t)=\frac{\mathrm{Ki} \cdot \mathrm{Mo}}{\mathrm{Ko}}\left(1-\mathrm{e}^{-\mathrm{Ko} \cdot \mathrm{t}}\right)
$$

and at equilibrium $(t=\infty)$

$$
\frac{\mathrm{Mi}(\infty)}{\mathrm{Mo}}=\frac{\mathrm{Ki}}{\mathrm{Ko}}
$$


Since $\mathrm{Mi}(\infty) / \mathrm{Mo}$ is not a constant (Table 2), either $\mathrm{Ki} / \mathrm{Ko}$ must vary and thus influx and efflux are not affected in the same way by changes in concentration, or not all of the methionine included in $\mathrm{Mi}(\infty)$ is available to exert its full chemical potential. It is difficult to define more clearly the kinetics of the exchange system until these alternatives are resolved.

Two models may be proposed to explain the uptake and exchange kinetics of the egg. A dual enzyme model would have an exchange system with a strict one-for-one transfer lying in parallel in the membrane with an active saturable accumulating system. A single enzyme model would require a system capable of transporting amino acids in various ratios, in the presence of a priming external concentration, responsible for both exchange and net accumulation. The latter model is preferred on several grounds. (i) It has a precedent in the L-system of the Erlich cell (Oxender \& Christiansen, 1963); (ii) a two-enzyme model would require a passive leak to offset net uptake at the equilibrium point and such a leak is not compatible with the lack of radioactivity leakage in amino acid-free medium at equilibrium; (iii) the substrate specificities of both exchange and uptake systems are the same; (iv) the equilibrium pool sizes at a wide range of external concentrations are not affected by temperature (unpublished), which could argue for identical effects of temperature on both influx and efflux kinetics, and (v) kinetic considerations suggest independent effects of substrate concentration on affinity (see above).

The balance of evidence therefore suggests, but does not prove, the presence in the egg membrane of a single transport system capable of transporting a class of large neutral amino acids in either direction across the membrane. The opposing fluxes may not be tightly linked and may show different responses to substrate concentration changes. The system appears to concentrate amino acid into an exchangeable pool which in turn may equilibrate more slowly with a non-exchangeable pool.

One very clear result does emerge from these experiments, and that is the close identity of the methionine-transporting system(s) in the egg before and after fertilization. It seems reasonable to assume that fertilization in the mouse does not result in the insertion or activation of increased numbers of amino acid-transporting enzymes of similar or novel kinetic activity, as appears to be the case in the sea urchin (Epel, 1972). In the mouse, such an alteration of transport activity occurs just before blastocyst formation (Borland \& Tasca, 1974) at the time of increase in rate of total protein synthesis (Brinster, 1971; Braude, 1979). However, some changes in the activity of the amino acid-transport system during cleavage may be inferred from a comparison of previously published kinetic data at the 4-cell stage (Borland \& Tasca, 1974) with that reported here for eggs before or after fertilization. At the 4-cell stage, uptake does not appear to come into equilibrium at longer incubation times (although a plot of the uptake data of Borland \& Tasca (1974) against time gives a straight line that does not go through the origin). This observation would suggest that a simple exchange system coming into equilibrium was no longer operating at the 4-cell stage. Absence or reduction of an efflux component could result from a change in enzyme function or because the substrate amino acid in the freely exchangeable pool was more effectively transferred to the non-exchangeable pool or incorporated into protein, thus becoming unavailable for exchange. If such a change in the kinetics of intercompartmental transfer of amino acid is indeed occurring, it becomes of considerable importance to know whether all of the protein-synthetic machinery of the cell draws equally on all available amino acid pools. In the absence of this knowledge, it would be possible (a) to draw misleading conclusions from incorporation data about changes in the rate of total protein synthesis during cleavage, (b) to misinterpret changes in the qualitative patterns of protein synthesis during cleavage, and (c) to misinterpret changes which might result not from new mRNA synthesis or activation, but to changes in the access of labelled amino acids to different protein synthetic compartments within the cell.

We acknowledge the excellent technical assistance of Miss Gin Flack, Miss Jo Close and Ms Debbie Eager. The work was supported by grants to M.H.J. from the Medical Research Council and the Ford Foundation. 


\section{References}

Borland, R.M. \& Tasea, R.J. (1974) Activation of a $\mathrm{Na}^{+}$-dependent amino acid transport system in preimplantation mouse embryos. Devl Biol. 36, 169182.

Borland, R.M. \& Tasca, R.J. (1975) $\mathrm{Na}^{+}$-dependent amino acid transport in preimplantation mouse embryos. Devl Biol. 46, 192-201.

Braude, P.R. (1979) Control of protein synthesis during blastocyst formation in the mouse. Devl Biol. (in press).

Brinster, R.L. (1971) Uptake and incorporation of amino acids by the preimplantation mouse embryo. $J$. Reprod. Fert. 27, 329-338.

Brinster, R.L., Wiebold, J.L. \& Brunner, S. (1976)
Protein metabolism in preimplantation mouse ova. Devl Biol. 51, 215-224.

Epel, D. (1972) Activation of a $\mathrm{Na}^{+}$dependent amino acid transport system upon fertilization of sea urchin eggs. Expl Cell Res. 72, 74-89.

Epstein, C.J. \& Smith, S.A. (1973) Amino acid uptake and protein synthesis in preimplantation mouse embryos. Devl Biol. 33, 171-184.

Oxender, D.L. \& Christiansen, H.N. (1963) Distinct mediating systems for the transport of neutral amino acids by the Ehrlich cell. J. biol. Chem. 238, 36863699.

Whittingham, D.G. (1971) Culture of mouse ova. $J$. Reprod. Fert., Suppl. 14, 7-22.

Received 19 September 1978 